\title{
Clinical Analysis of Targeted Navel Drops in the Treatment of Diabetes Mellitus
}

\author{
Yueping Zhu* \\ Wuxi Baylor Life Therapy, Wuxi 214100, Jiangsu Province, China \\ *Corresponding author: Yueping Zhu, 23905298796@126.com
}

\begin{abstract}
Objective: To analyze and evaluate the clinical efficacy of targeted navel drops in the treatment of diabetes mellitus. Methods: The time span of the study was from September 2020 to April 2021, and 288 diabetic patients were selected from our hospital. The patients were divided into research group $(n=96)$ group, control group $1(n=96)$ and control group $2(n=$ 96) by random number table. Control group 1 was managed with diet and exercise alone, control group 2 was treated with metformin on the basis of control group 1, and based on the treatment plan of the two control groups, the research group was treated with targeted navel drops. The relevant indexes of the three groups were compared and analyzed. Results: There was no significant difference in the fasting blood glucose and 2-hour postprandial blood glucose among the three groups $(p>0.05)$ before the treatment. After treatment, the fasting blood glucose and 2-hour postprandial blood glucose of the research group were significantly lower than those of control group 1 and control group $2(p<0.05)$. The total effective rate of the research group was $95.8 \%$, that of control group 1 was $85.4 \%$, and that of control group 2 was $79.2 \%$. The total effective rate of the research group was significantly higher than that of control group 1 and control group $2(p<0.05)$. Conclusion: There is a significant effect of targeted navel drops on diabetic patients in reducing their blood sugar levels, and its curative effect is better than using a single hypoglycemic therapy belonging to western medicine. It can be widely promoted at all levels in medical institutions.
\end{abstract}

Keywords: Targeted navel drops; Navel administration; Diabetes

Publication date: September 2021; Online publication: September 30, 2021

\section{Introduction}

The main pathological type of diabetes is type 2 diabetes, also known as non-insulin-dependent diabetes mellitus. In this type of diabetes, the body can normally form insulin but in vivo tissue cells are not able to establish an effective response to insulin; thus, the hormone is not able to play the clinical effect of regulating blood sugar. It can be concluded that the main pathological features of type 2 diabetes are insulin resistance and insulin secretion. At present, people's diet and daily life habits have changed significantly. The incidence rate of diabetes has increased year by year, and the disease is affecting the younger population. This disease has become a public health issue, requiring prompt and effective interventions. The conventional treatment for diabetes is hypoglycemic drugs belonging to western medicine, which can relieve clinical symptoms in short term. Its disadvantage is that there are multiple side effects from consuming these drugs. Hypoglycemia and other adverse events are easily inducible during treatment, resulting in a significant reduction in terms of treatment compliance. If only western medicines are used to control complications, the effect would be poor; thus, the treatment mode needs to be adjusted. According to the theory of traditional Chinese medicine, diabetes mellitus is under the category of diabetes. The treatment of diabetes with traditional Chinese medicine is a topic of interest in current clinical research ${ }^{[1]}$. Traditional Chinese medicine hypoglycemic drop is a diabetic treatment technique developed by our 
hospital. It can be used to repair damaged islet cells, reduce the incidence of various kinds of diabetic complications, effectively control blood sugar levels, and improve the quality of life of patients with diabetes mellitus by using traditional Chinese medicine, such as Dendrobium officinale, Artemisia annua, Ligusticum chuanxiong, Costus, Artemisia argyi, and Acorus calamus ${ }^{[2]}$. In order to further evaluate the clinical application value of this hypoglycemic navel drop, this study had selected diabetic patients in our hospital as the subjects and explored the clinical curative effect of the hypoglycemic navel drop.

\section{Data and methods}

\subsection{General information}

The time span of the study was from September 2020 to April 2021. A total of 288 diabetic patients were selected from our hospital. The patients were divided into the research group $(n=96)$ group, control group $1(\mathrm{n}=96)$, and control group $2(\mathrm{n}=96)$ by random number table. The basic clinical data of the three groups were summarized and analyzed. In the research group, there were 52 male patients and 44 female patients, age ranging from 51 years old to 77 years old, with an average age of $63.58 \pm 2.79$, and the statistical median of the course of disease was $4.46 \pm 1.07$ years. In control group 1, there were 54 male patients and 42 female patients, age ranging from 53 years old to 75 years old, with an average age of $63.46 \pm 2.88$, and the statistical median of the course of disease was $4.51 \pm 1.13$ years. In control group 2, there were 53 male patients and 43 female patients, age ranging from 52 years old to 76 years old, with an average age of 63.58 \pm 2.94 , and the statistical median of the course of disease was $4.69 \pm 1.12$ years. There was no significant difference in the comparative analysis of the basic data among the three groups $(p>0.05)$.

Inclusion criteria: (1) met the diagnostic criteria for WHO type 2 diabetes mellitus; (2) no drug allergies; (3) no critical complications; (4) informed consent given for this study.

Exclusion criteria: (1) complicated with kidney, heart, brain, and other critical complications; (2) drug allergies; (3) cognitive impairment and unable to cooperate in this study.

\subsection{Methods}

Diet and exercise intervention was implemented for patients in control group 1. The doctors made a diet plan for the patients according to their condition, distributed healthy recipes, and calculated the daily intake of calories, cholesterol, fat, and other substances. At the same time, the patients were instructed to exercise to improve their immunity for 4 weeks.

Based on the intervention for control group 1, control group 2 was treated with metformin for hypoglycemic treatment. The drug was given thrice daily, 30 minutes before meals, with a single dose of $0.5 \mathrm{~g}$ for 4 weeks.

Based on the treatment plan of the two control groups, the research group was treated with targeted navel drops. The drug components include Dendrobium candidum, Artemisia scoparia, Ligusticum chuanxiong, wood incense, wormwood leaf oil, Acorus tatarinowii, and other traditional Chinese medicine. Three to five drops were placed in the umbilicus, once in the morning and evening every day for a total of 4 weeks.

\subsection{Evaluation criteria}

The fasting blood glucose and 2-hour postprandial blood glucose of the three groups were compared and analyzed before and after treatment.

The therapeutic effects of the three groups were compared and analyzed. Cured was defined as a decrease of blood glucose index by more than $30 \%$ after treatment compared to before treatment or fasting blood glucose and 2-hour postprandial blood glucose lower than $7.2 \mathrm{mmol} / \mathrm{L}$ and $8.3 \mathrm{mmol} / \mathrm{L}$, respectively, 
with resolved clinical symptoms. The criteria to denote improvement were defined as blood glucose index decreased by 10-30\% after treatment compared to before treatment or fasting blood glucose lower and 2hour postprandial blood glucose lower than $8.3 \mathrm{mmol} / \mathrm{L}$ and $10.0 \mathrm{mmol} / \mathrm{L}$, respectively, with resolved clinical symptoms. The standard of ineffective was defined as no improvement in blood glucose and clinical symptoms after treatment. The cured and improved patients were deemed as effective therapeutic effect, and their proportion was counted.

\subsection{Statistical analysis}

Statistical Package for the Social Sciences (SPSS) version 23.0 software was used for statistical analysis of the data in this study. The measurement data conforming to normal distribution were represented by $(\bar{x} \pm \mathrm{s})$, t-test, and count data were represented by $(\%), \chi^{2}$ test. $P<0.05$ indicated statistically significant differences between the groups.

\section{Results}

\subsection{Comparison of fasting blood glucose and 2-hour postprandial blood glucose of the three groups before and after treatment}

There was no significant difference in the fasting blood glucose and 2-hour postprandial blood glucose among the three groups before treatment $(p>0.05)$. The fasting blood glucose and 2-hour postprandial blood glucose of the research group after treatment were significantly lower than those of control group 1 and control group $2(p<0.05)$ (Table 1).

Table 1. Comparison of fasting blood glucose and 2-hour postprandial blood glucose among the three groups before and after treatment $(\bar{x} \pm \mathrm{s}, \mathrm{mmol} / \mathrm{L})$

\begin{tabular}{cccccc}
\hline \multirow{2}{*}{ Group } & \multirow{2}{*}{$\begin{array}{c}\text { Number of } \\
\text { cases }\end{array}$} & \multicolumn{2}{c}{ Fasting blood glucose } & \multicolumn{2}{c}{ 2-hour postprandial blood glucose } \\
\cline { 3 - 6 } & 96 & $7.96 \pm 2.55$ & $6.01 \pm 1.68^{*}$ & $12.65 \pm 3.94$ & $7.06 \pm 1.46^{*}$ \\
\hline Research group & 96 & $7.75 \pm 2.44$ & $7.56 \pm 2.15$ & $11.93 \pm 3.71$ & $10.21 \pm 3.13$ \\
Control group 1 & 96 & $7.92 \pm 2.46$ & $7.18 \pm 1.96$ & $11.87 \pm 3.89$ & $9.58 \pm 2.91$ \\
Control group 2 & 96 & Bfter treatment & Before treatment & After treatment \\
\hline
\end{tabular}

Note: Compared with control group 1 and control group 2, the difference was significant, $* p<0.05$

\subsection{Comparison of the therapeutic effects among the three groups}

The total effective rate of the research group was $95.8 \%$, that of control group 1 was $85.4 \%$, and that of control group 2 was $79.2 \%$. The total effective rate of the research group was significantly higher than that of control group 1 and control group $2(p<0.05)$ (Table 2 and Table 3 ).

Table 2. Comparison of the therapeutic effects between the research group and control group 1 (n/\%)

\begin{tabular}{cccccc}
\hline Group & Number of cases & Cured & Improved & Ineffective & Total effective rate of treatment \\
\hline Research group & 96 & $78(81.3)$ & $14(14.6)$ & $4(4.2)$ & $92(95.8)$ \\
Control group 1 & 96 & $0(0.0)$ & $82(85.4)$ & $14(14.6)$ & $82(85.4)$ \\
$\chi 2$ value & & & & 6.350 \\
$p$ value & & & & 0.012 \\
\hline
\end{tabular}


Table 3. Comparison of the therapeutic effects between the research group and control group 2 (n/\%)

\begin{tabular}{cccccc}
\hline Group & Number of cases & Cured & Improved & Ineffective & Total effective rate of treatment \\
\hline Research group & 96 & $78(81.3)$ & $14(14.6)$ & $4(4.2)$ & $92(95.8)$ \\
Control group 2 & 96 & $0(0.0)$ & $76(79.2)$ & $20(20.8)$ & $76(79.2)$ \\
$\chi 2$ value & & & & & 12.597 \\
$p$ value & & & & 0.0. \\
\hline
\end{tabular}

\section{Discussion}

The main pathological feature of diabetes is chronic hyperglycemia. The pathogenic factors are insulin resistance, insulin secretion disorder, and so on. This disorder of the body is accompanied by protein, carbohydrate, and fat metabolism disorders. The extension of the course of the disease induces injuries to the kidneys, heart, blood vessels, nerves, and other systems. The main clinical treatment for diabetes includes hypoglycemic drugs belonging to western medicine and dietary control; such a program can correct hyperglycemia, but it does not remove the cause of diabetes or prevent its complications ${ }^{[3,4]}$.

According to traditional Chinese medicine, diabetes mellitus is under the category of diabetes. Its pathogenesis includes kidney yin deficiency, lung and stomach heat, as well as gas and Yin depletion. The treatment with hypoglycemic targeted navel drops is an external treatment method for diabetes, which has been developed by our hospital. Drugs can act on relevant acupoints drugs and can be quickly absorbed as well as utilized by the body through the navel. This is helpful in improving the efficacy of hypoglycemic drugs. The main composition of the traditional Chinese medicine hypoglycemic drop is Dendrobium candidum, which is the first Chinese medicinal herb. It is a type of traditional Chinese medicine. It has the effect of clearing away heat, nourishing Yin, promoting body fluid, and nourishing the stomach. It can remove the cause of diabetes, such as lung and stomach heat, gas and Yin consumption, and so on ${ }^{[5]}$. Wormwood, Ligusticum chuanxiong, wood incense, wormwood leaf oil, Acorus tatarinowii, and other auxiliary drugs are also compositions of the hypoglycemic drop. Among them, Ligusticum chuanxiong is a drug for Qi in blood, which has the effect of promoting blood circulation and regulating qi. Drugs such as Acorus tatarinowii and costus root are used for activating blood and regulating qi. Different prescriptions can eliminate the cause, repair damaged islet cells, prevent all kinds of complications, effectively control blood sugar, gradually reduce the dietary restriction of diabetics, and eventually improve their quality of life ${ }^{[6]}$.

\section{Conclusion}

In this study, the fasting blood glucose, 2-hour postprandial blood glucose, and the therapeutic effect of the patients in the research group were better than those of control group 1 and control group 2. Targeted navel drops have not been widely used in the treatment of diabetes. Doctors need to rationally select the prescription according to the patients' clinical manifestations, standardize all operations in the treatment, and observe whether there are adverse effects during the treatment in order to improve the therapeutic effect and the safety of the treatment. The comprehensive treatment of diabetes should be based on a variety of interventions. In addition to drug therapy, it is necessary to strengthen health education for patients, inform them of the importance of medication according to prescriptions from the doctors, explain matters related to the daily life of diabetics, guide them to avoid all pathogenic risk factors, and actively prevent all kinds of complications. At the same time, physicians need to provide diet guidance and healthy recipes to diabetic patients as well as inform them of the suitability of diabetic food so that they would be fully aware of the importance of a reasonable diet in managing diabetes and blood sugar control. In addition, doctors need to 
come up with exercise plans for these patients and guide them to gradually improve their immunity through exercise and walking so as to enhance the therapeutic effect. Some diabetic patients are not compliant to treatment. Therefore, doctors need to provide psychological comfort, encourage patients to actively cooperate with treatment interventions, and explain the therapeutic effects in order to achieve effective control of the disease.

To sum up, the use of targeted navel drops in diabetic patients has a significant effect on hypoglycemia and its curative effect is better than a single hypoglycemic therapy belonging to western medicine. It should be widely promoted at all levels in medical institutions. However, the total number of patients selected in this study was relatively small and the duration of this study was short. In addition to the lack of cross comparison research and analysis in the same type of research data in the region, the process design needs to be adjusted and the related mechanism of the use of navel drops in diabetic patients still requires continuous evaluation.

\section{Disclosure statement}

The author declares that there is no conflict of interest.

\section{References}

[1] Wang H, Yang Bo, Geng Y, et al., 2018, Yiqi Tongluo Decoction Combined with Thioctic Acid Injection in the Treatment of 60 Cases of Diabetic Peripheral Neuropathy with Qi Yin Deficiency and Blood Stasis Syndrome. Chinese Medicine Journal, 59(13): 1131-1134.

[2] Tian T, Pu X, Jin L, et al., 2018, Effect of Alprostadil Injection Combined with Shuxuetong Injection on Diabetic Nephropathy and Its Influence on Serum Inflammatory Factors, Hemorheology and Renal Function. Hebei Chinese Medicine, 40 6): 894-897.

[3] Cai F, Li C, 2019, Ligustrazine Injection in the Treatment of Diabetic Nephropathy. Medicine Frontier, 9(12): 206.

[4] Shi L, Zhang Z, Yuan X, et al., 2017, Dynamic Glucose Characteristics and Hypoglycemic Adjustment of Diabetic Patients After Glucocorticoid Application. Hainan Medicine, 28(9): 13941397.

[5] Li Q, Lin G, 2021, Combination of Traditional Chinese Medicine Packaging A-Lipoic Acid in the Treatment of Type 2 Diabetic Neurogenic Bladder. Jilin Medicine, 42(1): 158-160.

[6] Luo K, Zhang C, Chen L, et al., 2021, Clinical Effect of Chinese Medicine Foot Bath Combined with Traditional Chinese Medicine Package on Painful Diabetic Peripheral Neuropathy. Journal of Qiannan Medical College for Nationalities, 34(1): 8-11. 